\title{
Inhalt.
}

Einleitung. Selto

$\S$ 1. Studium und praktische Ausübung der Chirurgie . . . . . . . . . . 1

\$ 2. Geschichte der Chirurgie . . . . . . . . . . . . . . . . . . . 2

$\S$ 3. Entwickelung der modernen Chirurgie . . . . . . . . . . . . . . . 9

Erster Abschnitt. Allgemeine chirurgische Operationstechnik.

I. Ober die Vorbereitungen zu einer antiseptischen resp. aseptischen Operation.

§ 4. Definition einer chirurgischen Operation . . . . . . . . . . . . . . . . 19

$\$$ 5. Indikation und Kontraindikation einer Operation. . . . . . . . . . . . . . . . 19

$\S 6$. Vorbereitungen zu einer antiseptischen resp. aseptischen Operation. Antisepsis und Asepsis . . . . . . . . . . . . . . . . . . . . . . . 20

II. Die Schmerzstillung während der Operation. Die Narkose. Die lokale Anästhesie.

§ 7. Die Schmerzstillung wh̆hrend der Operation . . . . . . . . . . . . . 36

§ 8. Die Chloroformnarkose . . . . . . . . . . . . . . . . . . . . . 33

§ 9. Technik der Chloroformnarkose . . . . . . . . . . . . . . . . . 41

$\$$ 10. Symptomatologie der Chloroformnarkose. . . . . . . . . . . . . . 46

\$ 11. Üble Zufälle wăhrend der Chloroformnsrkose . . . . . . . . . . . . . . 48

8 12. Vorkommen und Ursachen des Chloroformtodes . . . . . . . . . . . . . 49

§ 13. Behandlung der üblen Zufälle während der Narkose. - Die übrigen Methylverbindungen . . . . . . . . . . . . . . . . . . . . . . 53

§ 14. Die Äthernarkose . . . . . . . . . . . . . . . . . . . . . . . . . . . . . . . . . 57

§ 15. Die Stickstoffoxydul- oder Lachgasnarkose . . . . . . . . . . . . . . . 63

§ 16. Gemischte Narkosen und sonstige Anăsthetika (Bromäthyl, Ätherbromat, Bromoform, Somnoform, Narkoform, Pental usw.) . . . . . . . . . 64

$\S$ 17. Die lokale Anästhesie. - Die Medullaranästhesie . . . . . . . . . . 67

III. Die Blutsparung bei den Operationen. Die kunstliche Blutleere nach v. Esmarch.

\$ 18. Die Blutsparung bei den Operationen . . . . . . . . . . . . . . 75

§ 19. Die künstliche Blutleere nach v. Esmarch . . . . . . . . . . . . . . . . 75

IV. Allgemeines Uber die Ausfluhrung einer aseptischen Operation und Uber die Nachbehandlung der Operierten.

§ 20. Ausführung einer aseptischen Operation. Antisepsis und Asepsis . . . . 81

今 21. Üble Zufälle während der Operation (Ohnmacht, Krämpfe, Blutung, Operation bei Blutern, Lufteintritt in die Venen, Tod) . . . . . . . . . . . 84

§ 22. Nachbehandlung der Operierten . . . . . . . . . . . . . . . . . . . . . . . . . 89

§ 23. Die wichtigsten Todesursachen nach Operationen . . . . . . . . . . . 90

\section{Die verschiedenen Methoden der Gewebstrennung.}

§ 24. Die blutige Trennung der Weichteile . . . . . . . . . . . . . . . . . 91

§ 25. Unblutige Trennung der Gewebe (Ligatur, Ekrasement, Glüheisen, Thermokauter, Galvanokaustik, Elektrolysc, Galvanopunktur, Ätzmittel usw.) . . 98

§ 26. Die Durchtrennung des Knochens . . . . . . . . . . . . . . . . 104 
VI. Die Blutstillung.

\$ 27. Die Blutstillung bei Operationen durch Unterbindung (Ligatur) der Gefaße 109

§ 28. Ersatzmittel der Ligatur . . . . . . . . . . . . . . . . . . . 114

8 29. Sonstige Blutstillungsmittel . . . . . . . . . . . . . . . . . . 116

\$ 30. Die Unterbindung der Arterien in der Kontinuität . . . . . . . . . 11

VII. Die Ableitung der Wundsekrete. Die Drainage der Wunden.

§ 31. Die Ableitung der Wundsekrete (Drainage der Wunden) . . . . . . . 121

VIII. Die Vereinigung der Gewebe. Die Wundnaht.

§ 32. Behandlung der Wunden vor Anlegung der Wundnaht . . . . . . . . 124

§ 33. Vereinigung der Weichteile. Die Wundnaht . . . . . . . . . . . 125

§ 34. Vereinigung der Knochenwundflächen . . . . . . . . . . . . . . 131

IX. Ober Amputationen, Exartikulationen und Resektionen.

§ 35. Allgemeines über die Ausführung der Amputationen und Exartikulationen . 133

§ 36. Methodik der Amputationen . . . . . . . . . . . . . . . . . . 134

\$ 37. Methodik der Exartikulationen . . . . . . . . . . . . . . . . . . . 142

8 38. Nachbehandlung der Amputationen und Exartikulationen. Nachkrankheiten 144

8 39. Prothesen nach Amputationen und Exartikulationen . . . . . . . . . 147

$\S$ 40. Die Operationen an den Gelenken. - Die Resektion, Arthrodese usw. . . 149

X. Ober die Operationen bei Gewebsdefekten. Plastische Operationen. Transplantation.

§ 41. Plastische Operationen bei Hautdefekten . . . . . . . . . . . . . 153

8 42. Die Hauttransplantation . . . . . . . . . . . . . . . . . . . . . . . . 159

8 43. Plastische Operationen an anderen Geweben (Sebnen, Muskeln, Nerven, Knochen). - Einheilung von Paraffin . . . . . . . . . . . . . 163

\section{Zweiter Abschnitt. Allgemeine chirurgische Verbandtechnik.}

\section{Die antiseptischen resp. aseptischen Wundverbände.}

§ 44. Allgemeines über antiseptische Wundverbände nach Lrstre. Antisepsis und Asepsis . . . . . . . . . . . . . . . . . . . . . . . . 165

§ 45. Die gebräuchlichsten antiseptischen resp. aseptischen Verbandstoffe . . . 166

\$ 46. Die verschiedenen Antiseptika . . . . . . . . . . . . . . . . . 168

\$ 47. Welche Antiseptika und welche antiseptische resp. aseptische Verband. methoden sind empfehlenswert? . . . . . . . . . . . . . . . 180

§ 48. Der antiseptische resp. aseptische Verbandwechsel . . . . . . . . . 184

II. Die sonstigen Wundverbănde resp. Wundbehandlungsmethoden.

§ 49. Sonstige Wundverbände . . . . . . . . . . . . . . . . . . . 187

III. Allgemeine Regeln uber die Anlegung von Binden und Verbandtuchern.

§ 50. Die Anlegung der Binden . . . . . . . . . . . . . . . . . . 192

$\S$ 51. Die Anlegung von Verbandtüchern . . . . . . . . . . . . . . . 200

IV. Die Lagerung der Kranken. Ober Lagerungsapparate und Lagerungsverbände.

§ 52. Die Lagerung der Kranken . . . . . . . . . . . . . . . . . . . 204

§ 53. Uber Lagerungsapparate, Schienen usw. - Heilgymnastik. Apparate nach ZANDER, Herz, TrENNERT u. a. . . . . . . . . . . . . . . . . 206

V. Die Technik der immobilisierenden Kontentivverbănde mit erhärtenden Stoffen und der Extensionsverbände.

§ 54. Die Technik der immobilisierenden Kontentivxerbănde mit erhärtenden Stoffen (Gips, Wasserglas usw.) . . . . . . . . . . . . . . . 218

8 55. Die Technik der Extensiongverbände . . . . . . . . . . . . . . 225 


\section{Dritter Abschnitt. Allgemeine chirurgische Pathologie und Therapie.}

I: Allgemeines Uber Entzlundung und Verletzung. Seite

§ 56. Allgemeines über Entzündung . . . . . . . . . . . . . . . . . 231

\$ 57. Ursachen der Entzündung . . . . . . . . . . . . . . . . . . . . . . . . . . . . . . . . 237

§ 58. Symptomatologie, Diagnose und Behandlung der Entzündung . . . . . . 239

\$ 59. Die Morphologie und allgemeine Bedeutung der Mikroorganismen (Mikroben) 252

\$60. Allgemeines über Verletzung . . . . . . . . . . . . . . . . . 278

§ 61. Die feineren anatomischen Vorgänge bei der Wundheilung. - Bildung der GefäBnarben („Organisation“ des Thrombus). - Die Blutgerinnung . . 281

§ 62. Allgemeine Reaktion nach der Verletzung und Entzündung. Die Lehre vom Fieber . . . . . . . . . . . . . . . . . . . . . . . 298

8 63. Shock. . . . . . . . . . . . . . . . . . . . . . . . . . 310

\& 64. Delirium tremens . . . . . . . . . . . . . . . . . . . . . 313

\$ 65. Delirium nervosum und psychische Störungen nsch Verletzungen . . . . 314

\& 66. Allgemeine Bemerkungen über die Wundinfektionskrankheiten . . . . . 315

\$ 67. Die Entzündung und Eiterung der Wunde. - Die wichtigsten Eitermikroben 318

8 68. Die akute Entzündüng der LymphgefäBe und Lymphdrüsen (Lymphangoitis, Lymphadenitis) . . . . . . . . . . . . . . . . . . . . . 326

§ 69. Die Entzündung der BlutgefäBe (Arteriitis und Phlebitis) . . . . . . . . 328

§ 70. Die Phlegmone . . . . . . . . . . . . . . . . . . . . . . . . . . 330

\$ 71. Erysipelas (Rotlauf, Rose). - Zoonotisches Erysipeloid (S. 348) . . . . . . 339

\& 72. Hospitalbrand (Wunddiphtherie) . . . . . . . . . . . . . . . . . . 348

\& 73. Der Wundstarrkrampf (Trismus, Tetanus) . . . . . . . . . . . . . . . $\quad 350$

8 74. Die Septikâmie. - Der Wundscharlach (S. 366). - Anhang: Die Pest (S. 367) 359

§ 75. Die Pyamie . . . . . . . . . . . . . . . . . . . . . . . 370

\$ 76. Infektion durch Leichengift . . . . . . . . . . . . . . . . . . 375

\& 77. Der Milzbrand (Anthrax). - Der Rauschbrand (S. 385) . . . . . . . . . . 377

\& 78. Der Rotz oder Wurm (Malleus) . . . . . . . . . . . . . . . . . . . 386

8. 79. Die Maul- und Klauenseuche (Aphthae epizooticae) . . . . . . . . . . . 390

\$ 80. Die Wut (Hydrophobie, Lyssa, Rabies) . . . . . . . . . . . . . . . . . 392

\$ 81. Vergiftungen durch Insekten, Schlangen usw. - Die japanische RattenbiBkrankheit . . . . . . . . . . . . . . . . . . . . . . . . 397

§ 82. Vergiftung der Wunden durch das Pfeilgift der Indianer und sonstiger wilder Völker . . . . . . . . . . . . . . . . . . . . . . 400

Anhang.

Chronische Mykosen: Tuberkulose (Skrofulose), Syphilis, Lepra, Aktinomykose.

\$ 83. Die Tuberkulose. - Die Skrofulose (S. 416) . . . . . . . . . . . . 401

\& 84. Die Syphilis (Lues) . . . . . . . . . . . . . . . . . . . . . . . . . . . . . . . 417

\$ 85. Die Lepra (der Aussatz) . . . . . . . . . . . . . . . . . . . . . . . . . 432

\$ 86. Die Aktinomykose . . . . . . . . . . . . . . . . . . . . . . . . . . . . . 439

II. Die Verletzungen und chirurgischen Erkrankungen der Weichteile.

\$ 87. Die Wunden der Weichteile. . . . . . . . . . . . . . . . . . 446

\$ 88. Behandlung der Weichteilwunden. - Sehnen- und Nervennaht. Sehnentransplantation . . . . . . . . . . . . . . . . . . . . .

89. Behandlung der Folgezustände nach gröBeren Blutverlusten (Bluttransfusion, Infusion von Kochsalzlösung). . . . . . . . . . . . .

§ 90. Verbrennung. - Einwirkung des Blitzes (S. 484). - Verbrennung durch Sonnenstrahlen (S. 486). - Der Sonnenstich (S. 486). Der Hitzschlag (S. 487)

8 91. Die Einwirkung der Kălte (Erfrierung) \& $_{\text {92. Subkutane Verletzung der Weichteile (Quetschung, Kontusion, subkutane }}$ ZerreiBung und Luxation der Muskeln, Sehnen und Nerven usw.) . . . 493

8 93. Die Entzündungen resp. Erkrankungen der Haut und des Zellgewebẹs . . 503

8 94. Die Entzündungen resp. Erkrankungen der Schleimhăute . . . . . . . 520

8 95. Die Entzündungen resp. Erkrankungen der Blutgefaße (Aneurysmen, Varicen usw.) . . . . . . . . . . . . . . . . . . . . . . . . . . . . 524

\& 96. Die Entzündungen resp. Erkrankungen des Lymphgefä8ystems . . . . . 537 
Erkrakungen der peripheren Nerven

98. Die Erkrankungen der periphen Nom

\& 98. Die Erkrankungen der Muskeln, Sehnen und Sebnenscheiden . . . . . $\quad 543$

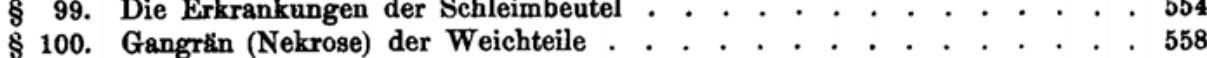

III. Die Verletzungen und Krankheiten der Knochen.

\& 101. Die Knochenbrïche (Frakturen); Vorkommen, Entstehnng und verschiedene Arten derselben . . . . . . . . . . . . . . . . . . . . . 563

8 102. Symptomatologie und klinischer Verlauf, Diagnose und Prognose der Frakturen . . . . . . . . . . . . . . . . . . . . . . . 574

\$ 103. Behandlung der Frakturen . . . . . . . . . . . . . . . . . . 590

\& 104. Uber Knochenquetschungen und Knochenwunden . . . . . . . . . . 609

\& 105. Die Entzündungen der Knochen . . . . . . . . . . . . . . . . 610

\& 106. Die aküten Entzündungen der Knochen; akute Periostitis und akute Osteomyelitis ... . . . . . . . . . . . . . . . . . . . . . . 611

§. 107. Die chronischen Entzündungen der Knochen; chron. Periostitis, Ostitis, Osteomyelitis; Tuberkulose, Syphilis der Knochen usw. . . . . . . . 620

\$ 108. Die Nekrose der Knochen . . . . . . . . . . . . . . . . . . . . . 632

\& 109. Spontane Epiphysenlösung . . . . . . . . . . . . . . . . . . . . . 639

\& 110. Rachitis (Zwiewuchs, englische Krankheit) . . . . . . . . . . . . 639

\& 111. Die Osteomslacie . . . . . . . . . . . . . . . . . . . . . . . . . . 647

§ 112. Atrophie und Hypertrophie der Knochen. - Riesenwuchs. - Akromegalie. - Akromikrie. - Tägliche Größenschwankungen. - Lymphadenia ossium

8 113. Die parasitären Geschwülste dẹr Knochen (Echinococcus und Cysticercus cellulosae) . . . . . . . . . . . . . . . . . . . . . . . . 658

IV. Die Verletzungen unid Krankheiten der Gelenke.

\& 114. Anatomische Vorbemerkungen über Gelenke . . . . . . . . . . . 661

\& 115. Die akuten Entzündungen der Gelenke . . . . . . . . . . . . . . . . 663

\& 116. Die chronischen Entzündungen der Gelenke. - Gelenkkrankheiten bei

Blutern. - Gelenkhämorrhagien bei Hirnläsionen . . . . . . . . . 673

8 117. Von den Gelenkkörpern (Mures articulares) . . . . . . . . . . . . . . 693

8 118. Nervöse (hysterische) Gëlenkkrankheiten, Neuralgien (Neurosen) der Gelenke 697

8 119. Neuropathische Knochen- und Gelenkaffektionen . . . . . . . . . . 699

\$ 120. Die Ankylosen . . . . . . . . . . . . . . . . . . . . . . . . . . . . 703

8 121. Die Deformitäten der Gelenke (Kontrakturen). - Schlottergelenke . . . 706

\& 122. Die Verletzungen der Gelenke. - Die Kontusion der Gelenke . . . . . 714

$\S$ 123. Die Distorsion der Gelenke . . . . . . . . . . . . . . . . . 716

8 124. Die Luxationen der Gelenke . . . . . . . . . . . . . . . . . 718

§ 125. Die Wúnden der Gelenke . . . . . . . . . . . . . . . . . . 731

Anhang.

§ 126. Über SchuBverletzungen. Kriegschirurgische Bemerkungen . . . . . . 783

V. Die Lehre von den Geschwulsten.

8 127. Allgemeines über Geschwülste . . . . . . . . . . . . . . . . . . 752

§ 128. Ätiologie der Geschwülste . . . . . . . . . . . . . . . . . . . . . . . 753

$\S$ 129. Wachstum, klinischer Verlauf, Diagnose und allgemeine Behandlung der Geschwülste

§ 130. Die verschiedenen Arten der Geschwülste. Geschwülste der Bindesubstanzen (Fibrom, Myхom, Lipom, Chondrom, Osteom, Angiom, Lymphangiom, Myom, Neurom, Gliom, Lymphom, Sarkom, Endotheliom uвw.) . . . . 764

8 131. Die epithelialen Geschwülste (Papillom, Epitheliom, Adenom, Karzinom usw.) 802 \$ 132. Über Cysten, Balggeschwülste und Teratome (Dermoide, Hautanhänge usw.) 826

Register. . . . . . . . . . . . . . . . . . . . 833 